\author{
E. Rojas • B. El-Bennich • J. P. B. C. de Melo \\ M. Ali Paracha
}

\title{
Insights into the Quark-Gluon Vertex from Lattice QCD and Meson Spectroscopy
}

Received: 30 September 2014 / Accepted: 5 August 2015 / Published online: 21 August 2015

(C) Springer-Verlag Wien 2015

\begin{abstract}
By comparing successful quark-gluon vertex interaction models with the corresponding interaction extracted from lattice-QCD data on the quark's propagator, we identify common qualitative features which could be important to tune future interaction models beyond the rainbow ladder approximation. Clearly, a quantitative comparison is conceptually not simple, but qualitatively the results suggest that a realistic interaction should be relatively broad with a strong support at about $0.4-0.6 \mathrm{GeV}$ and infrared-finite.
\end{abstract}

\section{Introduction}

The study of the nonperturbative quark-gluon vertex has been of great interest over the past decade [1-15] and even though its tensor structure is well understood, a long way remains to determine the corresponding form factors. Complete solutions of the Dyson-Schwinger equations (DSE) for the quark-gluon vertex are so far out of reach, though tractable calculations can be realized by introducing model-dependent form factors [16-21]. One exciting feature of the DSE is that they provide a bridge between phenomenological models and the basic objects of quantum field theories, namely the Green functions which can be calculated with lattice-QCD simulations.

In the Rainbow-Ladder (RL) truncation of the DSE, in which the vertex structure is dictated by the perturbative limit, many hadronic properties of light mesons $(\lesssim 1 \mathrm{GeV})$, quarkonia and the nucleon have successfully been described [20]. This suggests that to some extent the simple RL vertex structure is sufficient to calculate masses and decay constants of flavorless mesons when a judicious interaction model or dressing function is employed. Direct extraction of the dressed quark-gluon vertex functions from lattice simulations is not straight forward, as form factors associated with the longitudinal and transverse components have been obtained in lattice simulations for different kinematic configurations, yet the range of space-like momenta on the lattice is rather limited and their use in numerical DSE applications is currently impracticable. However, in computing

Presented by E. Rojas at LIGHT-CONE 2014, May 26-30, NCSU-USA.

E. Rojas $(\varangle)$ · B. El-Bennich · J. P. B. C. de Melo · M. A. Paracha

Laboratorio de Física Teórica e Computacional, Universidade Cruzeiro do Sul, São Paulo, Brazil.

E-mail: rojas4000@yahoo.com

E. Rojas

Instituto de Física, Universidad de Antioquia, Calle 70 No. 52-21, Medellín, Colombia.

B. El-Bennich

Instituto de Física Teórica, Universidade Estadual Paulista, São Paulo, SP, Brazil.

M. A. Paracha

Department of Physics, School of Natural Sciences, National University of Science and Technology, Islamabad, Pakistan. 
the quark two-point function, lattice simulations do include, at least partially in quenched calculations, the effect of nonperturbative quark-gluon dressing. We made use of this in Ref. [11], where we extracted an effective quark-gluon vertex by numerical inversion of the quark DSE using lattice data for its dressed mass function; see Eq. 3 below.

In the present study, we also make use of an effective coupling strength, $g_{\text {eff }}^{2}$, which under certain circumstances allows to extract important qualitative information from lattice data, and compare it with the corresponding effective interactions of phenomenological models in RL approximation [22-26]. The DSE for the quark propagator and arbitrary flavor is given by,

$$
S^{-1}(p)=Z_{2}\left(i p+m^{\mathrm{bm}}\right)+Z_{1} g^{2} \int_{k} D^{\mu \nu}(q) \frac{\lambda^{a}}{2} \gamma_{\mu} S(k) \frac{\lambda^{a}}{2} \Gamma_{\nu}(k, p),
$$

where $q=p-k, \int_{k} \equiv \int_{0}^{\Lambda} \frac{d^{4} k}{(2 \pi)^{4}}$ denotes a Poincaré invariant regularization and $Z_{1}$ and $Z_{2}$ are the vertex and quark wave-function renormalization constants. In Landau gauge, the gluon propagator is transverse,

$$
D_{\mu \nu}(q)=T_{\mu \nu}(q) \Delta\left(q^{2}\right),
$$

with the projection operator $T_{\mu \nu}(q):=g_{\mu \nu}-q_{\mu} q_{\nu} / q^{2}$. In QCD, the quark-gluon vertex satisfies the SlavnovTaylor-Ward Identity (STWI) which can be well approximated by a "ghost-improved" Ball-Chiu (BC) vertex $[11,27-29]$ :

$$
\tilde{\Gamma}_{\mu}=\tilde{X}_{0}\left(q^{2}\right) F\left(q^{2}\right) \Gamma_{\mu}^{\mathrm{BC}}(k, p),
$$

where $\tilde{X}_{0}\left(q^{2}\right)$ and $F\left(q^{2}\right)$ are vertex and ghost dressing functions [11], respectively, and $\Gamma_{\mu}^{\mathrm{BC}}$ is the (BC) vertex [30]. In the RL approximation and Landau gauge, the gluon and vertex dressing functions can be absorbed into the definition,

$$
Z_{1} g^{2} D_{\mu \nu}(q) \Gamma_{\mu}(k, p) \longrightarrow \frac{\mathcal{G}\left(q^{2}\right)}{q^{2}} T_{\mu \nu}(q) \gamma_{\mu},
$$

and the effective coupling is the sum of two terms which describe the nonperturbative and perturbative regimes of the interaction [31]:

$$
\frac{\mathcal{G}\left(q^{2}\right)}{q^{2}}=\frac{8 \pi^{2}}{\omega^{4}} D \exp \left(-\frac{q^{2}}{\omega^{2}}\right)+\frac{8 \pi \gamma_{m} \mathcal{F}\left(q^{2}\right)}{\ln \left[\tau+\left(1+q^{2} / \Lambda_{\mathrm{QCD}}^{2}\right)^{2}\right]} .
$$

In Eq. (5), $\gamma_{m}=12 /\left(33-2 N_{f}\right), N_{f}=4, \Lambda_{\mathrm{QCD}}=0.234 \mathrm{GeV} ; \tau=e^{2}-1$; and $\mathcal{F}\left(q^{2}\right)=[1-$ $\left.\exp \left(-q^{2} / 4 m_{t}^{2}\right)\right] / q^{2}, m_{t}=0.5 \mathrm{GeV}$.

The combined framework of DSE and Bethe-Salpeter equations (BSE) has been successfully used to describe the pseudoscalar and vector meson spectrum, related weak decay constants and electromagnetic form factors [20]. For flavored observables with light-quark current masses, $q=u, d, s$, this continuum QCD approach successfully reproduces the semi-leptonic decays $K^{+} \rightarrow \pi^{0} \ell^{+} \nu_{\ell}$ and $K^{0} \rightarrow \pi^{-} \ell^{+} \nu_{\ell}$ which proceed via a flavor changing charged current with a propagating spectator quark in the impulse approximation [32-34]. The same is not true for heavy-to-light transition form factors and in general for heavy-light meson observables where one relies more on modeling [35-37]. In such cases, an improvement of the theoretical framework is direly needed [38-41]. The matrix element $\left\langle\pi^{0}(p)\left|\bar{s} \gamma_{\mu} u\right| K^{+}(k)\right\rangle$ can be used to determine physical observables such as branching fractions and the weak CKM matrix element $V_{u s}$. The transition amplitude for the said decay can be completely described by two Lorentz vectors $\left(t=Q^{2}\right)$,

$$
\left\langle\pi^{0}(p)\left|\bar{s} \gamma_{\mu} u\right| K^{+}(k)\right\rangle=f_{+}(t) K_{\mu}+f_{-}(t) Q_{\mu},
$$

where $K_{\mu}=(p+k)_{\mu}$ and $Q_{\mu}=(k-p)_{\mu}$ are, respectively, the sum and difference of the momenta of the initial and final-state mesons, $t=-Q^{2}$ and $f_{ \pm}(t)$ are scalar transition form factors.

Part of the DSE-BSE program is concerned with the calculation of hadronic observables and meson spectroscopy by means of a single quark-gluon interaction vertex. In order to accomplish this program, it is necessary and important to identify phenomenologically successful vertex models beyond the RL approximation. Our primary interest in this work is to provide some qualitative criteria in the construction of these models. 
Table 1 Mass spectrum and decay constants for flavor-singlet and nonsinglet $J^{P}=0^{-}$mesons, where we follow Particle Data Group conventions [43]

\begin{tabular}{llll}
\hline & Model 1 & Model 2 & Reference \\
\hline$m_{\pi}$ & 0.138 & 0.153 & $0.139[43]$ \\
$f_{\pi}$ & 0.139 & 0.189 & $0.1304[43]$ \\
$m_{\pi(1300)}$ & 0.990 & 1.414 & $1.30 \pm 0.10[43]$ \\
$f_{\pi(1300)}$ & $-1.1 \times 10^{-3}$ & $-8.3 \times 10^{-4}$ & $0.493[43]$ \\
$m_{K}$ & 0.493 & 0.541 & $0.156[43]$ \\
$f_{K}$ & 0.164 & 0.214 & $1.460[43]$ \\
$m_{K(1460)}$ & 1.158 & 1.580 & $2.984[43]$ \\
$f_{K(1460)}$ & -0.018 & -0.017 & $3.639[43]$ \\
$m_{\eta_{c}(1 S)}$ & 3.065 & 3.210 & 3.784 \\
$m_{\eta_{c}(2 S)}$ & 3.402 & &
\end{tabular}

Both models refer to the interaction ansatz in Ref. [31], where we use the values $\omega=0.4 \mathrm{GeV}$ and $\omega D=(0.8 \mathrm{GeV})^{3}$ for Model 1 and $\omega=0.6 \mathrm{GeV}$ and $\omega D=(1.1 \mathrm{GeV})^{3}$ for Model 2. Dimensioned quantities are reported in $\mathrm{GeV}$ and reference values in the last column include experimental averages

\section{Effective Coupling Strength}

It was shown in Ref. [11] that it is possible, within error bars, to reproduce lattice-QCD data [42] on the quark propagator with the ghost-improved BC vertex of Eq. (3). It is also well known that in solving the DSE numerically, the BC vertex can be approximated by $\frac{1}{2}[A(k)+A(p)] \gamma_{\mu}$ such that the solutions for $A\left(p^{2}\right)$ and $B\left(p^{2}\right)$ are similar to those employing the full $\mathrm{BC}$ vertex. This prompts us to compare our ghost-improved vertex model with common RL models focusing on effective coefficient of the vertex structure $\gamma_{\mu}$ in the DSE kernel. In this approximation, we can define the effective coupling strength $g_{\text {eff }}^{2}(q, p)$ in either case, RL or BC, as in Eq. (4),

$$
Z_{1} g^{2} D_{\mu \nu}(q) \Gamma_{\mu}(k, p) \longrightarrow \frac{g_{\text {eff }}^{2}\left(q^{2}\right)}{q^{2}} T_{\mu \nu} \gamma_{\mu}
$$

which corresponds to

$$
\frac{g_{\text {eff }}^{2}(q, p)}{q^{2}}= \begin{cases} & \mathcal{G}\left(q^{2}\right) \quad[\mathrm{RL}] \\ & 4 \pi \alpha_{s}(\mu) \Delta\left(q^{2}\right) F\left(q^{2}\right) \tilde{X}_{0}(A(k)+A(p)) / 2 \quad[\mathrm{BC}] \\ \approx & 4 \pi \alpha_{s}(\mu) \Delta\left(q^{2}\right) F\left(q^{2}\right) \tilde{X}_{0} .\end{cases}
$$

In Eq. (8), we made use of the heavy-flavor approximation, $\frac{1}{2}[A(k)+A(p)] \approx 1$, in the BC vertex, so that $g_{\text {eff }}^{2}\left(q^{2}\right)$ only depends on $q^{2}$. It is not worth introducing this factor as part of the effective coupling strength, since it is flavor dependent and this approximation can be seen to be reasonable from Fig. 3 of Ref. [11]. In there, the variation, $1 \lesssim(A(k)+A(p)) \frac{1}{2} \lesssim 1.5$, in the range, $(0.14 \mathrm{GeV})^{2} \lesssim p^{2} \lesssim(4.3 \mathrm{GeV})^{2}$, is for the light flavors whereas for heavy quarks this factor is close to one. The difference in the effective coupling strength between $\omega=0.4 \mathrm{GeV}$ and $\omega=0.8 \mathrm{GeV}$ is huge; thus, roughly speaking our conclusions are independent of these less significant details.

Despite the qualitative nature of our analysis, we may derive some insight on the necessary domain of support of the interaction, i.e. the combined effect of gluon- and vertex-dressing functions, from comparison with the meson spectroscopy produced by the interaction models and the interaction we extracted from the lattice simulation data, i.e. both ansätze in Eq. (8). From Table 1 it is clear that for $\omega=0.6 \mathrm{GeV}$ and $D \omega=(1.1 \mathrm{GeV})^{3}$ the mass values of the exited states $\pi(1300), K(1460)$ and $\eta_{c}(2 S)$ are in better agreement with the average experimental data than for $\omega=0.4 \mathrm{GeV}$. However, the interaction with $D \omega=(1.1 \mathrm{GeV})^{3}$ and $\omega=0.6 \mathrm{GeV}$ does not yield acceptable decay constants and overestimates the masses of the ground states. A better interaction candidate may be found in the choice $\omega=0.6 \mathrm{GeV}$ and $D=(0.8 \mathrm{GeV})^{3} / \omega$, yet we encounter numerical complications for these parameter values when we solve the DSE in the complex plane. ${ }^{1}$ For the time being this does impede the computation of the masses of radial resonances. Nonetheless, this problem can be overcome by using a Nakanishi representation of the propagators. The choice $\omega=0.6 \mathrm{GeV}$ is also motivated

\footnotetext{
1 The quark propagators exhibits pairs of complex conjugate singularities which complicate the calculation.
} 

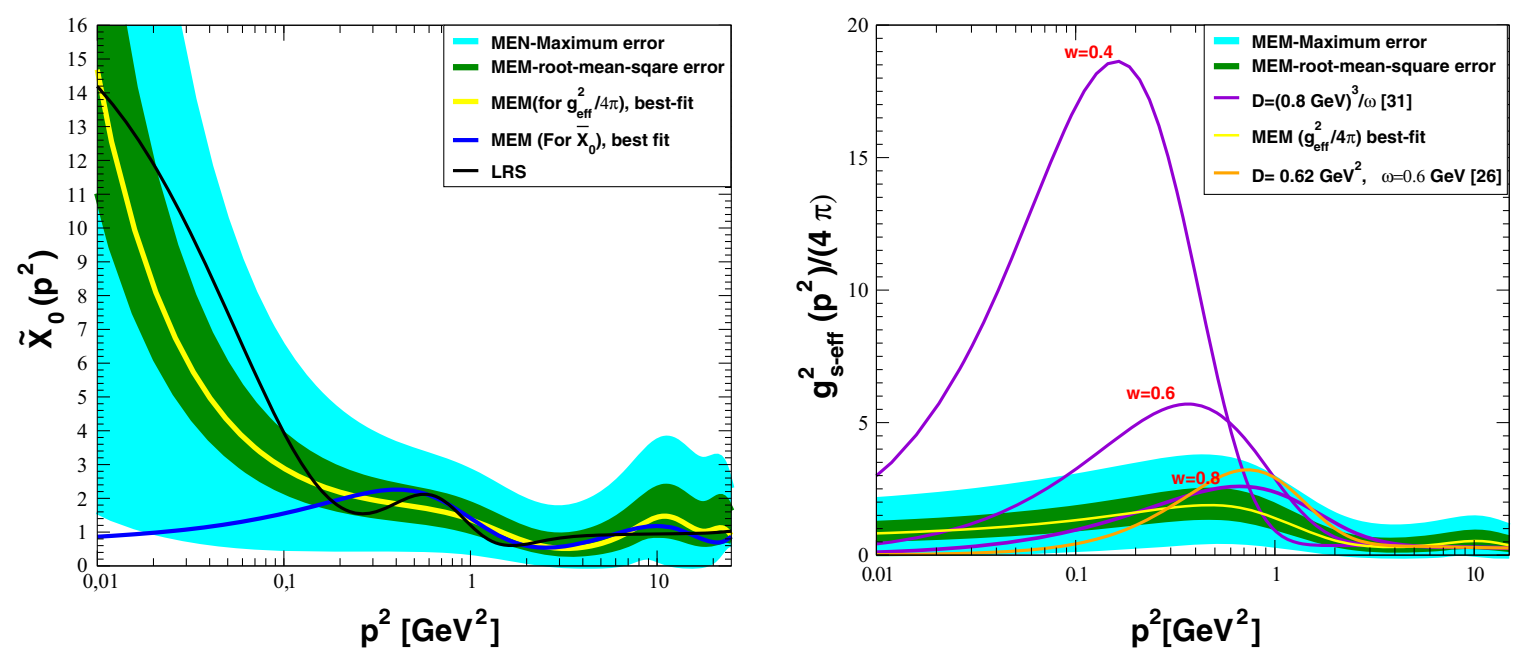

Fig. 1 Left panel the effective quark-gluon dressing form factor, $\tilde{X}_{0}$, computed using the maximum entropy method (MEM) and linear regularization (LRS); see Ref. [11] for details. The blue and yellow curves correspond to maximizing either the entropy of $\tilde{X}_{0}\left(p^{2}\right)$ or $g_{\text {eff }}^{2}\left(p^{2}\right) / 4 \pi$ in the MEM, where $g_{\text {eff }}^{2}\left(p^{2}\right)$ is the effective coupling strength of the interaction [29]. The latter is infrared enhanced, as in this case $p^{2} \tilde{X}_{0}\left(p^{2}\right)$ is finite in the limit $p^{2} \rightarrow 0$. Right panel the purple curves correspond three different parametrizations of the effective coupling strength $g_{\text {eff }}^{2}\left(p^{2}\right)=\mathcal{G}$ with the interaction model in Ref. [31] $\left(\omega D=(0.8 \mathrm{GeV})^{3}\right.$ in all cases); the orange curve corresponds to the effective coupling strength in the Maris-Tandy model [26]; the yellow curve corresponds to our effective coupling, $g_{\text {eff }}^{2}\left(p^{2}\right)=4 \pi \alpha_{s}(\mu) \Delta\left(p^{2}\right) F\left(p^{2}\right) \tilde{X}_{0}\left(p^{2}\right)$, with $\alpha_{s}(\mu)=0.295$ [29]

by the fact that the Maris-Tandy model [26] adequately reproduces experimental bottonium spectroscopy with the interaction parameters tuned to $D=0.62 \mathrm{GeV}^{2}$ and $\omega \sim 0.6 \mathrm{GeV}$ [44]. This is particularly significant since for heavy flavorless mesons the RL approximation is appropriate and flavor effects in the effective coupling strength should be minimal in this case.

In Fig. 1, we depict the effective coupling strength of Ref. [31] for three values of $\omega$ and $D=(0.8 \mathrm{GeV})^{3} / \omega$ fixed. In there, some common qualitative features may be appreciated; note in particular that the effective interaction is finite in the infrared. On the other hand, meson spectroscopy does not allow to draw general conclusions about the finiteness of the interaction. Modern DSE and lattice-QCD studies find that the gluon propagator is a bounded, regular function of spacelike momenta, which achieves its maximum value on this domain at $q^{2}=0$ [15]. Nonetheless, it is necessary to establish whether the vertex possesses some structure which can qualitatively alter this behavior as the effective interaction depends on the product of gluon propagator and vertex dressing functions. In general, it is quite difficult to put absolute constraints on $D \omega$ in the range $\omega \sim(0.4-0.8) \mathrm{GeV}$. However, once $D \omega$ is fixed the best values for the masses of the radial resonances correspond to a broader distribution in momentum space or equivalently larger values of $\omega$ [45]. Taking also into account that a broader distribution with a less pronounced peak is also required to reproduce the heavy-quarkonia spectrum, this suggests that a suitable interaction for a wide range of applications beyond the RL ought to have a strong and broad support concentrated at about $0.2-0.3 \mathrm{GeV}^{2}$.

A deeper insight is gained from the lattice data by inspection of the functional behavior of $\tilde{X}_{0}\left(q^{2}\right)$. All the solutions for $\tilde{X}_{0}$ in Fig. 1 are consistent with the lattice data for $A\left(p^{2}\right)$ and $B\left(p^{2}\right)$ as can be seen in Fig. 13 of Ref. [11]. However, it also becomes clear from Fig. 14 in the same reference that only when one solves the quark DSE with an infrared enhanced form factor $\tilde{X}_{0}$, the values of $A\left(p^{2}\right)$ and $B\left(p^{2}\right)$ become consistent with lattice QCD data at low momenta. This suggests an inflection of $\tilde{X}_{0}$ towards a finite limit for $q^{2} \rightarrow 0 \mathrm{GeV}^{2}$. Therefore, even though lattice data for the dressed-quark wave and mass functions do not strongly enough constrain the infrared behavior of $\tilde{X}_{0}\left(q^{2}\right)$ in the DSE inversion process, we find that requiring consistency between the DSE solutions and lattice QCD results provides a valuable source of information and constraints on the infrared domain.

\section{Conclusions}

By comparing experimental data on the pseudoscalar meson mass spectrum with realistic model calculations in the RL approximation, one deduces that the data prefer a broader distribution in momentum space for the 
effective coupling strength [45-47], $g_{\text {eff }}^{2}$, rather than a localized and sharply peaked interaction. An effective interaction extracted from lattice QCD data on the quark propagator is consonant with such a distribution and also points at an infrared-finite limit of $g_{\text {eff }}^{2}$. Self-consistency requires thus an infrared-finite functional behavior of $\tilde{X}_{0}$. Nonetheless, in order to go a step further in finding an interaction ansatz valid for a wide range of mesonic observables, one may need to resort to a more realistic quark-gluon-vertex interaction and take into account the important structure of its transverse components. In the past two decades, some progress in this direction has been made $[11,41,48-52]$ and phenomenological models should be extended to beyond the RL approximation using the guidelines of these studies as well as other input, such as the one proposed in the present study.

Acknowledgments This work was supported in part by the Brazilian agencies FAPESP (Fundação de Amparo à Pesquisa do Estado de São Paulo) and CNPq (Conselho Nacional de Desenvolvimento Científico e Tecnológico). We benefited from access to the computing facility of the Centro Nacional de Supercomputação at the Federal University of Rio Grande do Sul (UFRGS). We thank T. Frederico and O. Oliveira for their valuable collaboration. E. R. thanks financial support provided by "Sostenibilidad-UDEA 20142015" and the organizer of the Light-Cone 2014 Conference for the invitation.

\section{References}

1. Davydychev, A.I., Osland, P., Saks, L.: Quark gluon vertex in arbitrary gauge and dimension. Phys. Rev. D 63, 014022 (2001) [hep-ph/0008171]

2. Skullerud, J., Kizilersu, A.: Quark gluon vertex from lattice QCD. JHEP 0209, 013 (2002) [hep-ph/0205318]

3. Skullerud, J.I., Bowman, P.O., Kizilersu, A., Leinweber, D.B., Williams, A.G.: Nonperturbative structure of the quark gluon vertex. JHEP 0304, 047 (2003) [hep-ph/0303176]

4. Bhagwat, M.S., Holl, A., Krassnigg, A., Roberts, C.D., Tandy, P.C.: Aspects and consequences of a dressed quark gluon vertex. Phys. Rev. C 70, 035205 (2004) [nucl-th/0403012]

5. Bhagwat, M.S., Tandy, P.C.: Quark-gluon vertex model and lattice-QCD data. Phys. Rev. D 70, 094039 (2004) [hep-ph/0407163]

6. Skullerud, J.I., Bowman, P.O., Kizilersu, A., Leinweber, D.B., Williams, A.G.: Quark-gluon vertex in arbitrary kinematics. Nucl. Phys. Proc. Suppl. 141, 244 (2005) [hep-lat/0408032]

7. Matevosyan, H.H., Thomas, A.W., Tandy, P.C.: Quark-gluon vertex dressing and meson masses beyond ladder-rainbow truncation. Phys. Rev. C 75, 045201 (2007) [nucl-th/0605057]

8. Kizilersu, A., Leinweber, D.B., Skullerud, J.I., Williams, A.G.: Quark-gluon vertex in general kinematics. Eur. Phys. J. C 50, 871 (2007) [hep-lat/0610078]

9. Alkofer, R., Fischer, C.S., Llanes-Estrada, F.J., Schwenzer, K.: The quark-gluon vertex in Landau gauge QCD: Its role in dynamical chiral symmetry breaking and quark confinement. Annals Phys. 324, 106 (2009) [arXiv:0804.3042 [hep-ph]]

10. Hopfer, M., Windisch, A., Alkofer, R.: The quark-gluon vertex in landau gauge QCD. PoS Confinement X 073, (2012) [arXiv:1301.3672 [hep-ph]]

11. Rojas, E., de Melo, J.P.B.C., El-Bennich, B., Oliveira, O., Frederico, T.: On the quark-gluon vertex and quark-ghost kernel: combining lattice simulations with Dyson-Schwinger equations. JHEP 1310, 193 (2013) [arXiv:1306.3022 [hep-ph]]

12. Williams, R.: The quark-gluon vertex in Landau gauge bound-state studies. Eur. Phys. J. A 51(5), 57 (2015) [arXiv:1404.2545 [hep-ph]]

13. Gomez-Rocha, M., Hilger, T., Krassnigg, A.: First look at heavy-light mesons with a dressed quark-gluon vertex. arXiv:1408.1077 [hep-ph]

14. He, H.X., Liu, Y.X.: A dynamical mechanism for generating quark confinement. arXiv:1307.4485 [hep-ph]

15. Binosi, D., Chang, L., Papavassiliou, J., Roberts, C.D.: Bridging a gap between continuum-QCD and ab initio predictions of hadron observables. Phys. Lett. B 742, 183 (2015) [arXiv:1412.4782 [nucl-th]]

16. Roberts, C.D., Williams, A.G.: Dyson-Schwinger equations and their application to hadronic physics. Prog. Part. Nucl. Phys. 33, 477 (1994) [hep-ph/9403224]

17. Alkofer, R., von Smekal, L.: The Infrared behavior of QCD Green's functions: Confinement dynamical symmetry breaking, and hadrons as relativistic bound states. Phys. Rept. 353, 281 (2001) [hep-ph/0007355]

18. Maris, P., Roberts, C.D.: Dyson-Schwinger equations: a tool for hadron physics. Int. J. Mod. Phys. E 12, 297 (2003) [nucl-th/0301049]

19. Fischer, C.S.: Infrared properties of QCD from Dyson-Schwinger equations. J. Phys. G 32, R253 (2006) [hep-ph/0605173]

20. Bashir, A., Chang, L., Cloet, I.C., El-Bennich, B., Liu, Y.X., Roberts, C.D., Tandy, P.C.: Collective perspective on advances in Dyson-Schwinger Equation QCD. Commun. Theor. Phys. 58, 79 (2012) [arXiv:1201.3366 [nucl-th]]

21. Eichmann, G., Williams, R., Alkofer, R., Vujinovic, M.: The three-gluon vertex in Landau gauge. Phys. Rev. D 89 (10), 105014 (2014) [arXiv:1402.1365 [hep-ph]]

22. Munczek, H.J., Nemirovsky, A.M.: The Ground State q anti-q Mass Spectrum in QCD. Phys. Rev. D 28, 181 (1983)

23. Frank, M.R., Roberts, C.D.: Model gluon propagator and pion and rho meson observables. Phys. Rev. C 53, 390 (1996) [hep-ph/9508225]

24. Maris, P., Roberts, C.D., Tandy, P.C.: Pion mass and decay constant. Phys. Lett. B 420, 267 (1998) [nucl-th/9707003]

25. Maris, P., Roberts, C.D.: Pi- and K meson Bethe-Salpeter amplitudes. Phys. Rev. C 56, 3369 (1997) [nucl-th/9708029]

26. Maris, P., Tandy, P.C.: Bethe-Salpeter study of vector meson masses and decay constants. Phys. Rev. C 60, 055214 (1999) [nucl-th/9905056] 
27. Aguilar, A.C., Papavassiliou, J.: Chiral symmetry breaking with lattice propagators. Phys. Rev. D 83, 014013 (2011) [arXiv: 1010.5815 [hep-ph]]

28. Aguilar, A.C., Binosi, D., Cardona, J.C., Papavassiliou, J.: Nonperturbative results on the quark-gluon vertex. PoS Confinement X 103 (2012) [arXiv:1301.4057 [hep-ph]]

29. El-Bennich, B., Rojas, E., Paracha, M.A., de Melo, J.P.B.C.: Towards flavored bound states beyond rainbows and ladders. AIP Conf. Proc. 1625, 80 (2014) [arXiv:1311.3449 [nucl-th]]

30. Ball, J.S., Chiu, T.W.: Analytic properties of the vertex function in gauge theories. 1. Phys. Rev. D 22, 2542 (1980)

31. Qin, S.x., Chang, L., Liu, Y.x., Roberts, C.D., Wilson, D.J.: Interaction model for the gap equation. Phys. Rev. C 84, 042202 (2011) [arXiv:1108.0603 [nucl-th]]

32. Ji, C.R., Maris, P.: K(13) transition form-factors. Phys. Rev. D 64, 014032 (2001) [nucl-th/0102057]

33. Maris, P., Tandy, P.C.: Electromagnetic transition form-factors of light mesons. Phys. Rev. C 65, 045211 (2002) [nucl-th/0201017]

34. Ivanov, M.A., Kalinovsky, Y.L., Maris, P., Roberts, C.D.: Semileptonic decays of heavy mesons. Phys. Lett. B 416, 29 (1998) [nucl-th/9704039]

35. El-Bennich, B., Ivanov, M.A., Roberts, C.D.: Flavourful hadronic physics. Nucl. Phys. Proc. Suppl. 199, 184 (2010) [arXiv:0910.4523 [nucl-th]]

36. El-Bennich, B., Roberts, C.D., Ivanov, M.A.: Heavy-quark symmetries in the light of nonperturbative QCD approaches. arXiv:1202.0454 [nucl-th]

37. Ivanov, M.A., Korner, J.G., Kovalenko, S.G., Roberts, C.D.: B- to light-meson transition form-factors. Phys. Rev. D 76, 034018 (2007) [nucl-th/0703094]

38. Ivanov, M.A., Kalinovsky, Y.L., Roberts, C.D.: Heavy meson observables via Dyson-Schwinger equations. hep-ph/0006189

39. El-Bennich, B., de Melo, J.P.B.C., Loiseau, B., Dedonder, J.-P., Frederico, T.: Modeling electromagnetic form-factors of light and heavy pseudoscalar mesons. Braz. J. Phys. 38, 465 (2008) [arXiv:0805.0768 [hep-ph]]

40. Souchlas, N.: Bethe-Salpeter dynamics and the constituent mass concept for heavy quark mesons. Phys. Rev. D 81, 114019 (2010)

41. Fischer, C.S., Williams, R.: Probing the gluon self-interaction in light mesons. Phys. Rev. Lett. 103, 122001 (2009) [arXiv:0905.2291 [hep-ph]]

42. Parappilly, M.B., Bowman, P.O., Heller, U.M., Leinweber, D.B., Williams, A.G., Zhang, J.B.: Scaling behavior of quark propagator in full QCD. Phys. Rev. D 73, 054504 (2006) [hep-lat/0511007]

43. Beringer, J. et al. [Particle Data Group Collaboration]: Review of particle physics (RPP). Phys. Rev. D 86, 010001 (2012)

44. Blank, M., Krassnigg, A.: Bottomonium in a Bethe-Salpeter-equation study. Phys. Rev. D 84, 096014 (2011) [arXiv:1109.6509 [hep-ph]]

45. Popovici, C., Hilger, T., Gomez-Rocha, M., Krassnigg, A.: On a new approach to meson phenomenology with the BetheSalpeter equation. arXiv:1407.7970 [hep-ph]

46. Blank, M.: Properties of quarks and mesons in the Dyson-Schwinger/Bethe-Salpeter approach. arXiv:1106.4843 [hep-ph]

47. Rojas, E., El-Bennich, B., de Melo, J.P.B.C.: Exciting flavored bound states. Phys. Rev. D 90(7), 074025 (2014) [arXiv:1407.3598 [nucl-th]]

48. Bender, A., Roberts, C.D., Von Smekal, L.: Goldstone theorem and diquark confinement beyond rainbow ladder approximation. Phys. Lett. B 380, 7 (1996) [nucl-th/9602012]

49. Eichmann, G., Alkofer, R., Cloet, I.C., Krassnigg, A., Roberts, C.D.: Perspective on rainbow-ladder truncation. Phys. Rev. C 77, 042202 (2008) [arXiv:0802.1948 [nucl-th]]

50. Fischer, C.S., Williams, R.: Beyond the rainbow: effects from pion back-coupling. Phys. Rev. D 78, 074006 (2008) [arXiv:0808.3372 [hep-ph]]

51. Qin, S.X., Chang, L., Liu, Y.X., Roberts, C.D., Schmidt, S.M.: Practical corollaries of transverse Ward-Green-Takahashi identities. Phys. Lett. B 722, 384 (2013) [arXiv:1302.3276 [nucl-th]]

52. Heupel, W., Goecke, T., Fischer, C.S.: Beyond rainbow-ladder in bound state equations. Eur. Phys. J. A 50, 85 (2014) [arXiv:1402.5042 [hep-ph]] 